\title{
O CONHECIMENTO ECOLÓGICO LOCAL DOS PESCADORES ARTESANAIS SOBRE OS ELASMOBRÂNQUIOS MARINHO-COSTEIROS NA APA DO DELTA DO PARNAÍBA, NORDESTE DO BRASIL
}

\section{The local ecological knowledge of artisanal fishermen on the marine-coastal elasmobranchs in the Parnaíba delta MPA, northeast of Brazil}

\author{
Georgia Maria de Oliveira Aragão ${ }^{1^{*}}$, Gilvana Pessoa de Oliveira², \\ Jorge Eduardo Kotas ${ }^{3}$, Henry Louis Spach ${ }^{4}$

\begin{abstract}
${ }^{1}$ Discente no Programa de Pós-Graduação em Sistemas Costeiros e Oceânicos, Universidade Federal do Paraná, bolsista Capes, End. Pontal do Paraná - PR, 83255-000, Universidade Federal do Paraná, Centro de Estudos do Mar. E-mail: georgia.aragao@gmail.com 64202-020. E-mail: gilvana@ufpi.edu.br

${ }^{3}$ Analista Ambiental, ICMBIO-CEPSUL, End. Av. Carlos Ely Castro, 195 - Centro, Itajaí - SC, 88301-445. E-mail: jorge.kotas@icmbio.gov.br

${ }^{4}$ Docente, Programa de Pós-Graduação em Sistemas Costeiros e Oceânicos, Universidade Federal do Paraná, bolsista Capes, End. Pontal do Paraná - PR, 83255-000, Universidade Federal do Paraná,

Centro de Estudos do Mar. E-mail: henry@ufpr.br
\end{abstract} \\ ${ }^{2}$ Docente, Universidade Federal do Piauí, End. Av. São Sebastião, nº 2819 - Nossa Sra. de Fátima, Parnaíba - PI,
}

\begin{abstract}
RESUMO
O conhecimento ecológico tradicional consiste na forma em que as populações tradicionais se apropriam e usam os recursos naturais. A partir desse conceito buscou-se interpretar o conhecimento e as percepções dos pescadores artesanais da APA do Delta do Parnaíba sobre aspectos ecológicos dos elasmobrânquios e a conservação desse grupo. Para atender ao objetivo, foram aplicadas 100 entrevistas semiestruturadas. Os resultados, a partir dos registros do conhecimento local, indicaram a ocorrência de oito espécies de raias e nove de tubarões. A pesca incidental de raias e tubarões ocorre durante todo o ano, inclusive de espécies ameaçadas. Apesar disso, os pescadores demonstraram entender sobre a importância da conservação desse grupo. Os dados obtidos indicaram associação entre o conhecimento ecológico tradicional e a literatura científica. Além disso, os dados sugerem a necessidade de uma intervenção imediata no que diz respeito à fiscalização dos desembarques e campanhas educativas na região, pois ocorre a captura de espécies amea-
\end{abstract}

Recebido em: 13/09/2018

Aprovado em: 05/09/2019

Publicado online em: $1^{\circ} . / 11 / 2019$ 
çadas (i.e. Ginglymostoma cirratum). O trabalho com comunidades tradicionais pode contribuir com pesquisas e gestão participativa da pesca.

Palavras-chave: povos tradicionais, tubarões, raias, extrativismo.

\begin{abstract}
Traditional ecological knowledge consists of the way in which traditional populations appropriate and use natural resources. Based on this, it was tried to interpret the knowledge and perceptions of artisanal fishermen from the Parnaiba Delta MPA about the ecological aspects of elasmobranchs and the conservation of this group. To comply the goal, 100 semi-structured interviews were applied. The results from the local knowledge records indicated the occurrence of eight species of rays and nine of sharks. Incidental fishing for rays and sharks occurs throughout the year, including endangered species. Despite this, the fishermen demonstrated to understand about the importance of the conservation of this group. The data obtained indicated an association between traditional ecological knowledge and the scientific literature. In addition, the data suggest the need for immediate intervention in the surveillance of landings and educational campaigns in the region, as the capture of endangered species (i.e., Ginglymostoma cirratum) occurs. The work with traditional communities can contribute to research and participatory management of fisheries.
\end{abstract}

Keywords: traditional people, sharks, rays, extractivism.

\title{
INTRODUÇÃO
}

Diversas metodologias de pesquisa são utilizadas para conhecer a biodiversidade de elasmobrânquios em uma determinada região. Neste caso, o estudo do conhecimento ecológico tradicional (CET) auxilia especialmente no caso de espécies de difícil acesso (i.e., espécies ameaçadas de extinção). Populações humanas nativas em áreas costeiras são fonte potencial de informações acerca do ecossistema e suas funções (Abreu et al., 2017; Diegues \& Moreira, 2001; Freitas et al., 2017). Para nos aproximar das percepções dessas comunidades, podemos utilizar da etnobiologia, ciência que estuda o homem e as relações ambientais em seu cotidiano. A etnobiologia pode ser definida como o "estudo do conhecimento e das conceituações desenvolvidas por qualquer sociedade a respeito da biologia" (Posey, 1987). Com origens também na antropologia cognitiva, investiga "como o mundo é percebido, conhecido e classificado por diversas culturas humanas" (Begossi, 1993).

A APA Delta do Parnaíba possui uma área de 313.809 ha e abriga um delta com elevada biodiversidade e importância ecológica e econômica para os estados do Piauí e Maranhão (ICMBIO, 2009). A atividade econômica nessa área é apoiada pela prática extrativa, sendo representada pelos vários tipos de pescarias: peixes, camarão, siri e mariscos, e pela coleta do caranguejo-uçá (Farias et al., 2015). Os autores ainda discutem sobre a riqueza natural desse ecossistema em termos de produtividade primária, que tem origem principal no aporte e na capacidade de retenção de nutrientes, oriundos das bacias continentais, em função da extensa faixa de manguezais sustentada pela plataforma continental que é vasta e rasa.

A fauna de elasmobrânquios da APA do Delta do Parnaíba é explorada incidentalmente pelas pescarias de pequeno porte, que, de acordo com Dulvy et al. (2014), são orga- 
nismos que não fazem parte das espécies-alvo. Os tubarões e as raias são capturados principalmente por espinhéis, linhas de mão e redes de emalhe, apetrechos utilizados com a finalidade de capturar outras espécies, como é o caso do camurupim Megalops atlanticus (Valenciennes, 1847).

Considerando o atual declínio das espécies de raias e tubarões e sua relevante importância para a manutenção de processos ecológicos, especialmente em áreas de berçário (Dias-Neto, 2011), e a complexidade das possíveis estratégias para recuperação de recursos pesqueiros, é possível inferir sobre diferentes perspectivas sobre o tema, como as percepções das comunidades pesqueiras em relação aos recursos. Nesse sentido, o presente artigo tem como objetivo investigar o conhecimento ecológico local (CEL) dos pescadores sobre os elasmobrânquios e sua visão sobre a conservação desse grupo na APA do Delta, contrastando esse conhecimento com informações científicas sobre a biologia das espécies e da necessidade de se trabalhar com ambas linhas de conhecimento para a conservação das espécies ameaçadas.

\section{MATERIAIS E MÉTODOS}

\section{Coleta e análise de dados}

As coletas foram realizadas em dois municípios da APA do Delta do Parnaíba:

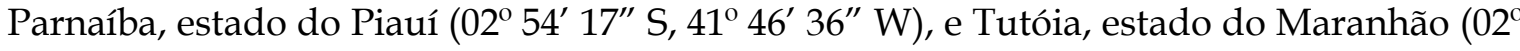
$45^{\prime} 43^{\prime \prime} \mathrm{S}, 42^{\circ} 16^{\prime} 28^{\prime \prime} \mathrm{W}$ ), onde estão localizados os principais pontos de desembarque pesqueiro em função do volume de captura (Figura 1).

A pesquisa se deu por meio de três etapas: 1) acompanhamentos semanais dos desembarques pesqueiros de setembro a novembro de 2015, com o objetivo de conhecer os aspectos gerais das comunidades, a atividade pesqueira, além de estabelecer vínculo com os pescadores; 2) entrevistas livres quinzenais, ou seja, conversas informais com os pescadores sobre as modalidades de pesca, espécies mais capturadas, espécies-alvo e que ocorreram de janeiro de 2016 a janeiro de 2017; 3) aplicação quinzenal de entrevista semiestruturada transcrita ipsis litteris por um pesquisador (o mesmo que realizou as entrevistas em todas as etapas) de janeiro a novembro de 2016.

A condição para que fosse aplicada a entrevista semiestruturada era de que os pescadores tivessem no mínimo 10 anos de atividade pesqueira para que se pudesse fazer a avaliação dos dados a partir de no mínimo 10 anos atrás. Os dados etnoecológicos foram coletados respeitando-se a identidade cultural local e estabelecendo uma relação mútua de confiança entre pesquisador e informantes, estabelecidas nas etapas 1 e 2 . Foi apresentado o Termo de Consentimento Livre e Esclarecido, também foram explicadas as vantagens e desvantagens da pesquisa e informado que não se faz obrigatório responder o que achar inconveniente.

O primeiro contato se deu por meio de um dos pescadores mais experientes da região, o qual indicou possíveis entrevistados e assim sucedeu até a última entrevista coletada, método conhecido como snowball (Albuquerque \& Lucena, 2004). Após consentimento prévio, entrevistas semiestruturadas foram aplicadas individualmente. A entrevista semiestruturada abordou questões sobre: I - Perfil Socioeconômico do Pescador; II - As Atividades Pesqueiras; III - Conhecimento Ecológico Tradicional e a Percepção Ambiental do Pescador.

A estatística descritiva foi utilizada para comparar as respostas dos entrevistados e os resultados foram apresentados como contagens (frequência absoluta) e percentagens 
(frequência relativa). Os dados obtidos das entrevistas também foram analisados qualitativamente sob uma perspectiva emic-etic (Posey, 1992), contrastando conhecimento e intenções da comunidade com o conhecimento acadêmico. Como complemento foi transcrita ipisis literis as respostas dos entrevistados.

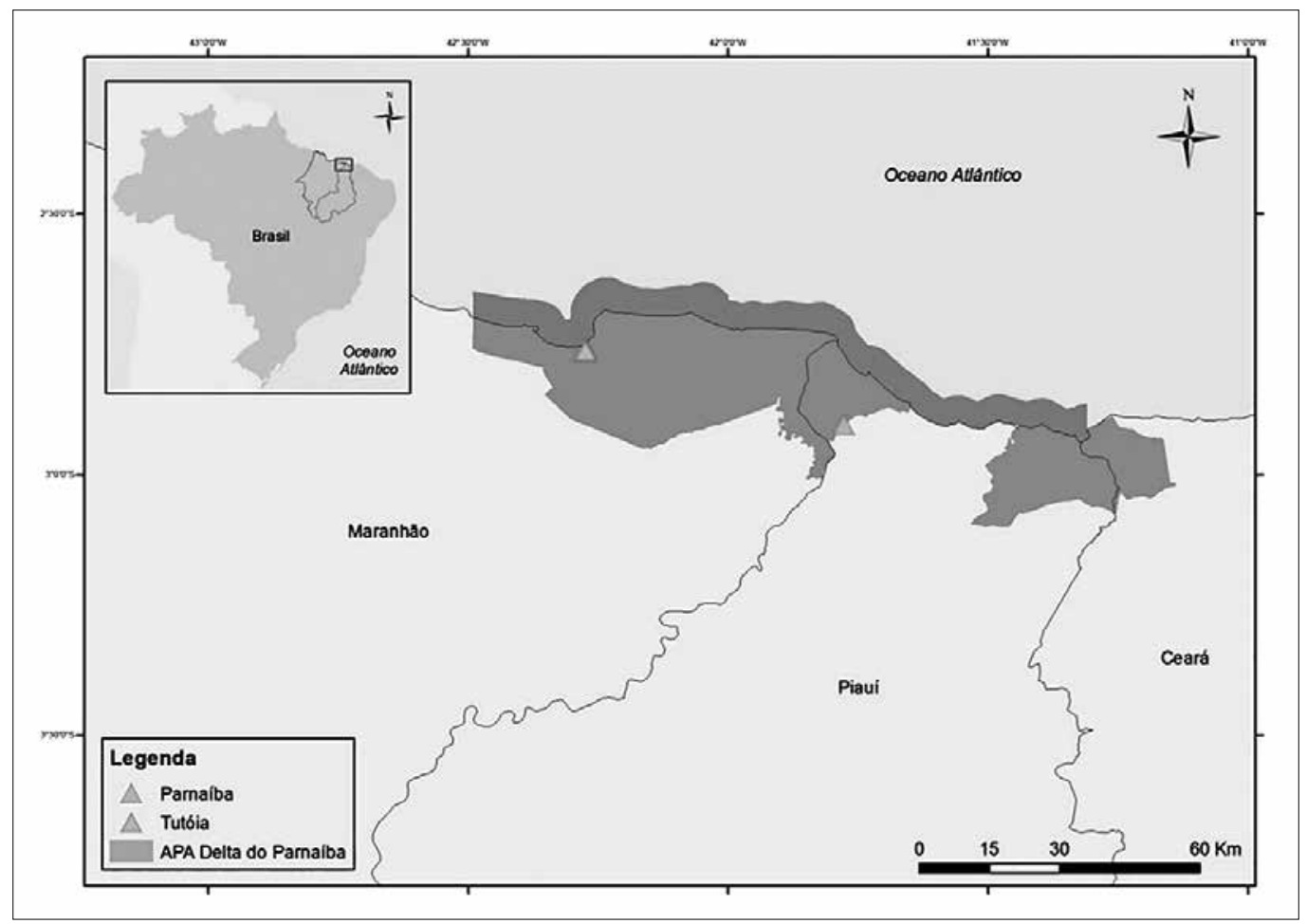

Figura 1 - Localização da APA do Delta do Parnaíba com os municípios de Parnaíba (PI) e Tutóia (MA) amostrados nas entrevistas

\section{RESULTADOS}

\section{Perfil socioeconômico}

Foram entrevistados 100 pescadores artesanais da APA do Delta do Parnaíba, dos quais 40 eram da localidade da Praia da Pedra do Sal, município de Parnaíba (PI), e 60 do município de Tutóia (MA).

No Piauí, 43\% dos pescadores entrevistados têm a idade entre 25 e 35 anos e, no Maranhão, 50\% têm entre 36 e 45 anos. Considerando a idade dos entrevistados e o tempo de pesca, nota-se em um primeiro momento que o conhecimento vem sendo passado através das gerações, tendo em vista que o tempo médio na pesca entre 10 e 20 anos representou $45 \%$ e $47 \%$ dos entrevistados no Piauí e Maranhão, respectivamente. A maioria tem apenas o ensino fundamental, ou seja, $65 \%$ dos entrevistados no Piauí e $53 \%$ no Maranhão. Quanto ao estado civil, 78\% dos piauienses e 77\% dos maranhenses são casados. Em ambos estados, $58 \%$ dos indivíduos têm a pesca como atividade econômica exclusiva, porém muitos realizam outras atividades (e.g. extrativismo e construção civil) (Tabela I). 
Tabela I - Perfil socioeconômico dos pescadores da APA do Delta do Parnaíba

\begin{tabular}{|c|c|c|c|c|}
\hline Idade & $\mathbf{n}_{\mathrm{PI}}$ & $\mathbf{n}_{\mathrm{MA}}$ & $\%$ PI & $\%$ MA \\
\hline $25-35$ & 17 & 19 & 43 & 32 \\
\hline $36-45$ & 16 & 30 & 40 & 50 \\
\hline $46-55$ & 4 & 8 & 10 & 13 \\
\hline$>55$ & 3 & 3 & 8 & 5 \\
\hline \multicolumn{5}{|l|}{ Escolaridade } \\
\hline Analfabeto & 14 & 20 & 35 & 33 \\
\hline Ensino fundamental & 26 & 32 & 65 & 53 \\
\hline Ensino médio & 0 & 8 & 0 & 13 \\
\hline \multicolumn{5}{|l|}{ Estado Civil } \\
\hline Solteiro & 9 & 14 & 23 & 23 \\
\hline Casado & 31 & 46 & 78 & 77 \\
\hline \multicolumn{5}{|l|}{ Tempo na pesca } \\
\hline $10-20$ & 18 & 28 & 45 & 47 \\
\hline $21-30$ & 16 & 22 & 40 & 37 \\
\hline $31-40$ & 3 & 6 & 5 & 10 \\
\hline$>40$ & 3 & 4 & 8 & 7 \\
\hline \multicolumn{5}{|l|}{ Atividade econômica } \\
\hline Pesca & 23 & 35 & 58 & 58 \\
\hline Pesca e extrativismo & 5 & 13 & 13 & 22 \\
\hline Pesca e construção civil & 6 & 6 & 15 & 10 \\
\hline Pesca e vigia & 1 & 2 & 3 & 3 \\
\hline Pesca e artesanato & 3 & 0 & 8 & 0 \\
\hline Pesca e aposentadoria & 2 & 4 & 5 & 7 \\
\hline
\end{tabular}

Nota: $\mathrm{n}_{\mathrm{PI}}=40$ e $\mathrm{n}_{\mathrm{MA}}=60$, onde $\mathrm{n}$ é a frequência absoluta e $\%$ percentual.

\section{Atividades pesqueiras e composição das capturas}

As artes de pesca mais utilizadas pelos pescadores artesanais maranhenses que atuam na APA do Delta do Parnaíba foram as seguintes: o espinhel (31\%), a linha de mão $(28 \%)$, rede de emalhe (26\%) e tarrafa $(15 \%)$. Por sua vez, para os pescadores piauienses, o espinhel $(100 \%)$, a rede de emalhe $(88 \%)$, a linha de mão $(75 \%)$ e a tarrafa $(13 \%)$ foram as mais utilizadas. Ressalta-se que ocorre a utilização de mais de um apetrecho de pesca por pescador entrevistado.

A pesca embarcada é realizada em grupos de duas a seis pessoas, sendo geralmente com até quatro pescadores (75\% PI e $88 \%$ MA). O único dia em que os grupos não saem para pescar é aos domingos. No Piauí, além do domingo, também não saem nos dias santos. O tempo de pesca varia de acordo com o apetrecho que está sendo utilizado, sendo o período com rede de emalhe o menor, com três horas em média, e a pesca com linha de mão o maior tempo, com 10 horas em média, como foi relatado por $100 \%$ dos pescadores entrevistados.

Quando questionados sobre a existência da pesca direcionada sobre as raias e cações, a maioria dos pescadores afirma não ocorrer (73\% PI e 65\% MA). De acordo com 68\% dos 
pescadores piauienses, apenas ocorre o descarte quando os animais são muito pequenos ou por não apresentarem valor econômico agregado, enquanto que os pescadores maranhenses (100\%) não descartam, levando para o consumo familiar.

A espécie de raia mais pescada na região é Hypanus guttatus (Bloch \& Schneider, 1801), popularmente conhecida como bicuda e que pode ser encontrada o ano todo, sendo esta espécie a mais apreciada comercialmente na região (Tabela II). Em relação aos tubarões, o mais pescado, de acordo com os piauienses (100\% dos entrevistados), é o rabo seco Rhizoprionodon porosus (Poey, 1861) enquanto para os maranhenses $(90 \%$ dos entrevistados) é o sacurí, Carcharhinus limbatus (Müller \& Henle, 1839). Ambos são registrados ao longo do ano. Os dados obtidos foram confrontados com a literatura existente (Tabela III).

Tabela II - Espécies de raias mais capturadas e épocas do ano em que são encontradas na APA do Delta do Parnaíba de acordo com as entrevistas realizadas com os pescadores artesanais das comunidades do Piauí e Maranhão e a literatura existente

\begin{tabular}{|c|c|c|c|c|c|}
\hline \multirow{2}{*}{$\begin{array}{l}\text { Espécies de raias } \\
\text { mais pescadas }\end{array}$} & \multirow{2}{*}{$\begin{array}{l}\% \text { PI } \\
(n=40)\end{array}$} & \multirow{2}{*}{$\begin{array}{l}\% \text { MA } \\
(n=60)\end{array}$} & \multicolumn{2}{|c|}{ Época do ano } & \multirow{2}{*}{ Literatura } \\
\hline & & & $\%$ PI & $\%$ MA & \\
\hline Hypanus guttatus & 100 & 100 & $88 \mathrm{AT}$ & $100 \mathrm{AT}$ & $\begin{array}{l}\text { Araujo \& Almeida, 2001; } \\
\text { Bigelow \& Shroeder, 1953; } \\
\text { Basilio et al., 2008; Lessa, } 1986\end{array}$ \\
\hline Hypanus americanus & 90 & 95 & $83 \mathrm{AT}$ & $79 \mathrm{AT}$ & $\begin{array}{l}\text { Bigelow \& Shroeder, 1953; Gadig } \\
\text { et al., 2000; Lessa, } 1986\end{array}$ \\
\hline Fontitrygongeijskesi & 88 & 90 & 94 ATC & 93 ATC & Lessa, 1986 \\
\hline Rhinopterabonasus & 73 & 83 & 90 ATS & 98 ATS & Gadig et al., 2000; Lessa, 1986 \\
\hline $\begin{array}{l}\text { Gymnura } \\
\text { micrura }\end{array}$ & 73 & 82 & $86 \mathrm{AT}$ & $78 \mathrm{AT}$ & Gadig et al., 2000; Lessa, 1986 \\
\hline Aetobatus narinari & 53 & 42 & $81 \mathrm{AT}$ & $88 \mathrm{AT}$ & Gadig et al., 2000; Lessa, 1986 \\
\hline
\end{tabular}

Nota: $\mathrm{n}$ = frequência absoluta, \% percentual por comunidade, Ano Todo - AT, Ano Todo com maior incidência em período de chuva - ATC, Ano Todo com maior incidência em período de seca - ATS.

Tabela III - Espécies de tubarões mais capturados e épocas do ano em que são encontradas na APA do Delta do Parnaíba de acordo com as entrevistas realizadas com os pescadores artesanais das comunidades do Piauí e Maranhão e a literatura existente

\begin{tabular}{|c|c|c|c|c|c|}
\hline \multirow[b]{2}{*}{$\begin{array}{l}\text { Espécie de tubarões } \\
\text { mais pescados }\end{array}$} & \multirow[b]{2}{*}{$\begin{array}{r}\% P I \\
(n=40)\end{array}$} & \multirow[b]{2}{*}{$\begin{array}{l}\% M A \\
(n=60)\end{array}$} & \multicolumn{2}{|c|}{ Época do ano } & \multirow[b]{2}{*}{ Literatura } \\
\hline & & & $\%$ PI & $\%$ MA & \\
\hline Rhizoprionodon porosus & 100 & 90 & $100 \mathrm{AT}$ & $100 \mathrm{AT}$ & $\begin{array}{l}\text { Almeida, 1998; Lessa, 1986; } \\
\text { Machado et al., 2000; Martins et } \\
\text { al., 2018; }\end{array}$ \\
\hline Sphyrna spp. & 100 & 83 & $100 \mathrm{AT}$ & $100 \mathrm{AT}$ & $\begin{array}{l}\text { Gadig et al., 2000; Lessa, 1986; } \\
\text { Martins et al., 2018; }\end{array}$ \\
\hline $\begin{array}{l}\text { Ginglymostoma } \\
\text { cirratum }\end{array}$ & 100 & 87 & $100 \mathrm{AT}$ & $100 \mathrm{AT}$ & $\begin{array}{l}\text { Gadig et al., 2000; Lessa, 1986; } \\
\text { Martins et al., 2018; }\end{array}$ \\
\hline Carcharhinus limbatus & 100 & 95 & $78 \mathrm{AT}$ & $93 \mathrm{AT}$ & Lessa, 1986; Martins et al., 2018; \\
\hline Carcharhinus porosus & 93 & 73 & $81 \mathrm{AT}$ & 89 AT & $\begin{array}{l}\text { Gadig et al., 2000; Lessa, 1986; } \\
\text { Martins et al., 2018; }\end{array}$ \\
\hline Carcharhinus acronotus & 88 & 80 & $86 \mathrm{AT}$ & $90 \mathrm{AT}$ & Lessa, 1986; Martins et al., 2018; \\
\hline
\end{tabular}

Nota: $\mathrm{n}$ = frequência absoluta, \% percentual por comunidade, 1, 2, 3, 4, 5, 6 correspondem às espécies com as épocas do ano, AT - Ano Todo. 
No que diz respeito ao tamanho médio dos elasmobrânquios pescados, todos os entrevistados disseram que o comprimento dos animais varia de acordo com a distância da costa, ou seja, quanto mais próximo, menor é o animal, e quanto mais longe, maior é o animal, principalmente os tubarões.

\section{Conhecimento ecológico tradicional e percepção ambiental}

De acordo com os dados das Tabelas IV e V, os pescadores da APA do Delta do Parnaíba veem a situação da pesca nos dias atuais como preocupante, ou seja, com chances de diminuir a pesca de espécies comerciais, como relatado pelos pescadores: "os peixes vão diminuir, já estão diminuindo e a culpa é de quem pesca os pequeno e de quem polui os ares" (relato do pescador 1 ).

Tabela IV - Percepção dos pescadores sobre a situação da pesca na APA do Delta do Parnaíba nos dias atuais e há 10 anos nas comunidades do Piauí e do Maranhão

\begin{tabular}{|c|c|c|c|c|}
\hline PERCEPÇÃO DOS PESCADORES* & $\begin{array}{l}\text { PI } \\
(n=40)\end{array}$ & $\begin{array}{l}\text { MA } \\
(n=60)\end{array}$ & $\%$ PI & $\%$ MA \\
\hline \multicolumn{5}{|l|}{ SITUAÇÃO DA PESCA NOS DIAS ATUAIS } \\
\hline Produção cada vez menor. & 31 & 49 & 38 & 32 \\
\hline Necessário ir pescar cada vez mais distante da costa. & 19 & 31 & 23 & 20 \\
\hline $\begin{array}{l}\text { Devido às mudanças climáticas, tem diminuído a produção de } \\
\text { peixe, quanto menos chuva, menos peixe. }\end{array}$ & 23 & 45 & 28 & 29 \\
\hline Peixes cada vez menores próximos à costa. & 8 & 29 & 10 & 19 \\
\hline \multicolumn{5}{|l|}{ COMO ERA A PESCA HÁ 10 ANOS } \\
\hline Maior diversidade de espécies. & 36 & 51 & 53 & 39 \\
\hline Menos tempo no mar pescando. & 10 & 32 & 15 & 25 \\
\hline Peixes maiores eram capturados. & 22 & 47 & 32 & 36 \\
\hline \multicolumn{5}{|l|}{$\begin{array}{l}\text { NAS PESCARIAS ATUAIS SE VÊ MAIS OU MENOS RAIAS E } \\
\text { CAÇÕES }\end{array}$} \\
\hline $\begin{array}{l}\text { Menos cações, pois pescamos os pequenos, aí eles não crescem } \\
\text { para "produzir". }\end{array}$ & 11 & 3 & 17 & 5 \\
\hline Algumas espécies sumiram como a viola e o espadarte. & 29 & 47 & 46 & 78 \\
\hline Das raias, algumas como a jamanta e a narim vêm diminuindo. & 23 & 10 & 37 & 17 \\
\hline
\end{tabular}

Nota: * mais de uma resposta por entrevistado, $\mathrm{n}$ frequência absoluta, \% percentual por comunidade.

Tabela V - Variáveis que medem a percepção dos pescadores sobre as espécies menos vistas no período entre 1996 e 2016 nas distintas comunidades

\begin{tabular}{lllll}
\hline Quais espécies são menos vistas nos últimos $\mathbf{1 0}$ anos & $\begin{array}{l}\text { PI } \\
(\mathbf{n = 4 0 )}\end{array}$ & $\begin{array}{l}\text { MA } \\
(\mathbf{n = 6 0 )}\end{array}$ & \% PI & \% MA \\
\hline Pseudobatos spp. & 40 & 51 & $25 \%$ & $31 \%$ \\
Mobula spp. & 26 & 19 & $16 \%$ & $11 \%$ \\
Sphyrna lewini & 38 & 34 & $23 \%$ & $20 \%$ \\
Pristis spp. & 40 & 55 & $25 \%$ & $33 \%$ \\
Galeocerdo cuvier & 18 & 7 & $11 \%$ & $4 \%$ \\
\hline
\end{tabular}

Nota: $\mathrm{n}$ frequência absoluta, \% percentual por comunidade.

Dos entrevistados, 38\% dos pescadores do Piauí e 32\% dos pescadores do Maranhão relatam que a produção está diminuindo, o que segundo eles pode estar associado às mudanças climáticas, pois a estiagem prolongada na região Nordeste está reduzindo as capturas (opinião de 28\% dos pescadores do PI e 29\% do MA). Para eles, há 10 anos a diversi- 
dade de peixes era maior (opinião de $53 \%$ dos pescadores do PI e 39\% do MA). Segundo os pescadores, algumas espécies de raias e tubarões não aparecem mais na região, como é o caso de Pseudobatos spp., que na região é chamado de cação viola, e do espadarte Pristis spp. (opinião de $46 \%$ dos pescadores do PI e 78\% do MA) (relato do pescador 2). Outras espécies, como a raia jamanta Mobula spp. e a narim Aetobatus narinari (Euphrasen, 1790), vêm diminuindo a incidência na região (opinião de $37 \%$ dos pescadores do PI e $17 \%$ do MA).

tem uns tipos de cação que a gente nem vê mais, tem gente aqui que nunca viu um espadarte e nem o cação viola. (Relato do pescador 2).

Quando questionados com a pergunta “Deve-se pescar menos, mesmo ganhando menos, para conservar alguma espécie de peixe?", a maioria responde que se existir alguma compensação financeira, sim (45\% dos entrevistados no PI e 65\% no MA), pois existe a necessidade de pescar mesmo que seja somente para consumo próprio (relato do pescador 3).

todo mundo precisa comer pra sobreviver, se tem peixe e não tem dinheiro a gente vai pegar o peixe. (Relato de pescador 3 ).

A maioria dos entrevistados concorda com os defesos para que determinadas espécies não diminuam sua abundância ou não entrem em extinção ( $58 \%$ dos entrevistados no PI e 67\% no MA). Muitos ainda citaram que é necessário parar de pescar principalmente os animais de menor porte, como os "cações de umbigo" (neonatos) (33\% dos entrevistados no PI e 30\% no MA) (relato do pescador 4). Por outro lado, 10\% dos pescadores entrevistados no Piauí e 3\% no Maranhão disseram "não ter jeito" para essa situação.

em nossa região tem um problema muito grande que é a pesca de cação de umbigo, se continuar assim os bichos vai sumir porque não tem como procriar, acaba mesmo. (Relato do pescador 4).

Porém, quando questionados sobre a importância das raias e tubarões para a vida marinha, somente 5\% dos entrevistados no Piauí e 3\% no Maranhão disseram que esses animais não têm importância alguma. Por outro lado, 12\% dos entrevistados no Piauí e $30 \%$ no Maranhão relataram que os animais são importantes para a renda e alimentação de suas famílias. No Maranhão, 55 \% dos entrevistados entendem a importância dos elasmobrânquios para o equilíbrio marinho e, no Piauí, 58\% acreditam que esses organismos servem para "limpar as águas", em especial os cações (Tabela VI e relato do pescador 5).

cada ser vivo tem uma função no mundo, a gente tem a nossa e os bicho do mar têm as deles e uma delas é alimentar a gente e comer os resto de bicho que ficam por aí, limpando as águas. (Relato do pescador 5).

Tabela VI - Variáveis que medem a percepção e o conhecimento ecológico dos pescadores sobre a importância dos elasmobrânquios nas distintas comunidades

\begin{tabular}{llcll}
\hline Importância das raias e dos cações para a vida marinha* & PI (n=40) & MA (n=60) & \% PI & \% MA \\
\hline São importantes para o equilíbrio marinho. & 11 & 33 & $26 \%$ & $55 \%$ \\
Eles limpam as águas, principalmente os cações. & 25 & 7 & $58 \%$ & $12 \%$ \\
Fonte de renda e alimentação para as famílias litorâneas. & 5 & 18 & $12 \%$ & $30 \%$ \\
Não têm importância. & 2 & 2 & $5 \%$ & $3 \%$ \\
\hline N
\end{tabular}

Nota: * mais de uma resposta por pescador, $\mathrm{n}$ frequência absoluta, \% percentual por comunidade. 


\section{DISCUSSÃO}

As espécies mais capturadas na APA do Delta do Parnaíba (H. guttatus, R. porosuse, Sphyrna spp.) são recorrentes na bibliografia da costa nordeste do Brasil (Araújo \& Almeida, 2001; Martins et al., 2018). Entretanto, no caso das espécies do gênero Sphyrna, são ameaçadas de extinção e, portanto, necessitam com urgência de medidas de conservação, o que implica em restrições à pesca (Kotas, 2004; Kotaset al. 2012). Isso corrobora com a situação relatada nas comunidades estudadas e comprova a diminuição populacional desses tubarões em suas atividades pesqueiras.

O conhecimento tradicional tem sido amplamente utilizado em estudos científicos para avaliar o estado de conservação de espécies marinhas (Sáenz-Arroyo \& RevolloFernández, 2016). Porém, poucos são os trabalhos realizados com foco nos elasmobrânquios, mesmo sendo este um meio de inferir sobre o nível de exploração dos organismos (Giglio \& BornatowskI, 2016; Reis-Filho et al., 2016; Feitosa et al., 2017). A integração do conhecimento tradicional e científico pode ser crucial para o entendimento de padrões biológicos e ecológicos, assim como fornecer informações de espécies de difícil acesso ou mesmo ameaçadas de extinção (Diegues, 2004).

Os sistemas de manejo dos recursos pesqueiros vêm sendo transmitidos através das gerações, mesmo que aconteça de forma adaptativa ao contexto contemporâneo, o qual sofre grandes pressões (Berkes, 1999). Isso pode ser verificado no presente estudo, onde o tempo de dedicação à atividade pesqueira variou de 10 a mais de 40 anos em ambas as comunidades estudadas, bem como ao apresentarem adaptações às pressões atuais, através da necessidade de atividades complementares de renda (e.g. extrativismo, construção civil, vigilância e artesanato).

A maioria dos pescadores da APA do Delta do Parnaíba é considerada artesanal, os quais têm como características a produção em pequena escala, baixo rendimento econômico, excedente reduzido e irregular e o domínio de conhecimentos adquiridos através das gerações (Batista et al., 2014; Diegues, 2004; Oliveira et al., 2016). A pesca artesanal apresenta contrastes com a pesca industrial, por ser exercida com métodos mais simples e diversificados, menor autonomia, ausência de tecnologias e menor produtividade (Begossi, 2004). Os resultados amostrados descrevem exatamente esse contexto, onde o uso do espinhel, redes de emalhe e anzol e linha são os mais utilizados pelos pescadores artesanais, apresentando baixa produtividade e pouca autonomia das embarcações. Apesar das pescarias na APA serem consideradas artesanais, elas podem ser impactantes sobre os elasmobrânquios, pelo fato de operarem em áreas mais costeiras, onde de uma maneira geral se encontram os principais berçários das espécies ameaçadas de extinção, como é o caso das espécies dos gêneros Isogomphodon e Sphyrna (Lessa et al., 2006; Baum et al., 2006).

Os pescadores entrevistados demonstraram possuir conhecimento sobre a sazonalidade e estrutura populacional das espécies de elasmobrânquios na região, como a presença de neonatos em épocas chuvosas e nas áreas rasas, a ocorrência dos indivíduos maiores em profundidades elevadas e sua relação com os fatores abióticos, como precipitação e distância da costa. Ressalta-se que as precipitações na região norte do Nordeste sofrem forte influência da Zona de Convergência Intertropical (Uvo \& Nobre, 1989). Entre esses fatores, a profundidade (Heithaus et al., 2007) e o tipo de fundo (Barbini, 2011) podem ser relacionados, quando relatam que os indivíduos maiores são pescados mais longe da costa e os menores mais próximos à costa. $\mathrm{O}$ conhecimento dos pescadores de que os fatores bióticos e abióticos estruturam a distribuição e abundância de espécies de elasmobrânquios também 
influencia na captura desses animais (Schlaff et al., 2014). Pode-se entender que o sucesso da pesca depende desse conhecimento, assim os pescadores sabem quando, onde e o que pescar. Entretanto, ocorre a captura dos cações de umbigo, bem como fêmeas grávidas, o que deveria ser evitado.

Os peixes são recursos explorados de acordo com a cultura própria de dada sociedade. No caso dos pescadores piauienses, os elasmobrânquios não fazem parte das espécies mais apreciadas e por isso não existe a pesca direcionada, pois os animais vêm como captura incidental (O’Riordan \& Turner, 1997). Porém, em Tutóia, existe uma forte cadeia de produtos derivados de elasmobrânquios, indicativo de um possível direcionamento a esse grupo (Martins et al., 2018). As espécies $R$. porosus e H. guttatus fazem parte dos principais recursos pesqueiros da pesca de pequena e média escala na região da APA do Delta do Parnaíba, a exemplo do que acontece para o restante do Nordeste brasileiro (Mendonça et al., 2011). Adicionalmente, Lessa (1986) e Almeida (1999) mostraram que R. porosus constitui uma parcela importante nos desembarques na costa do Maranhão, representando entre $18 \%$ e $45 \%$ da produção de elasmobrânquios provenientes da pesca costeira.

As espécies mais pescadas encontram-se com algum grau de risco de extinção (ICMBio, 2016). Embora $R$. porosus esteja classificada como Pouco Preocupante na lista da IUCN (Lessa et al., 2006), na lista brasileira esta espécie é considerada Criticamente em Perigo em decorrência da sua diminuição evidente nas capturas registradas no Brasil. Por sua vez, G. cirratum, que é a segunda espécie mais pescada na APA do Delta do Parnaíba, está categorizada como Dados Insuficientes na lista da IUCN (Rosa et al., 2006); contudo, na avaliação nacional é classificada como Vulnerável. Avaliações de estoques devem ser realizadas a nível regional para as diversas espécies categorizadas como Vulneráveis (VU), com o objetivo de fornecer subsídios técnicos para que planos de manejo sejam implementados para as mesmas, como têm sido feitas nas oficinas de avaliação de riscos de extinção das espécies. No caso das espécies ameaçadas (Em Perigo - EN e Criticamente Ameaçadas - CR), a sua proteção deverá ser integral, havendo a necessidade de planos de recuperação.

Os pescadores relataram que a raia-viola (Pseudobatos spp.), raia-jamanta (Mobula spp.), tubarão-martelo safroa (Sphyrna lewini) e espadarte, que configuram a lista de elasmobrânquios ameaçados, tiveram redução na frequência de captura na APA nos últimos dez anos. Por outro lado, com exceção do tubarão-tigre, que está próximo de ameaçado (NT), todas as outras se encontram em algum grau de ameaça (CR, EN, VU), segundo a IUCN red liste, a lista de espécies ameaçadas do ICMBio. Entretanto, diversos autores consideram que as avaliações da lista vermelha da IUCN devem ser periodicamente revistas, pois há espécies como A. narinari considerada quase ameaçada (NT) e várias espécies consideradas com dados deficientes (DD) e de menor preocupação (LC), que podem estar sofrendo níveis de ameaça mais críticos (Tagliafico et al., 2012; Dulvy et al., 2014).Também é importante salientar que várias espécies consideradas ameaçadas na lista vermelha global da IUCN não figuram com esse status na lista nacional e vice-versa, como é o caso de $R$. porosus (LC- globalmente) e CR (nacional). A exemplo, Bender et al. (2012) mostram em seu estudo comparativo de listagens de espécies de peixes recifais que $84,8 \%$ das espécies globalmente ameaçadas não aparecem na lista nacional, enquanto 54,5\% das espécies mencionadas com esse status na lista brasileira não estão na Lista Vermelha da IUCN.

Os pescadores da APA do Delta do Parnaíba têm conhecimento sobre os fatores que envolvem a diminuição das espécies pescadas e de sua biodiversidade, também sabem que a pesca de animais jovens e neonatos é um problema na conservação, estes últimos denominados por eles de "cações de umbigo", e que a sua captura contribui para a redução das 
populações de elasmobrânquios regional e globalmente. Além disso, reconhecem que produção está cada vez menor e por isso necessitam ir pescar em regiões mais distantes da costa. Concomitante à diminuição das capturas nessas áreas, eles também percebem que está ocorrendo a sobrepesca de neonatos, jovens e fêmeas em fase reprodutiva, comprometendo o recrutamento de elasmobrânquios na região (Dias-Neto, 2011).

Os relatos mencionaram que existe a necessidade de pescar, mesmo que seja somente para consumo próprio. Portanto, é válido o que Castello (2008) diz sobre a viabilidade da preservação dos recursos pesqueiros no Brasil estar relacionada com a necessidade de consolidar um modelo de gestão que leve em consideração as características da pesca e as necessidades humanas. Nesse sentido, os modelos de gestão devem ser baseados no etnoconhecimento que beneficiem a manutenção da biodiversidade e a diversidade cultural (Diegues, 2000).

Os estoques de elasmobrânquios estão cada vez menores ao longo de todos os oceanos (Barbosa-Filho et al., 2014; Dulvy et al., 2017) e parte expressiva dos pescadores da APA do Delta do Parnaíba não acredita existir reversão para esse cenário negativo. Porém, para a maioria dos entrevistados, os períodos de defeso e áreas de proibição da pesca poderiam ser uma possível solução. Entretanto, Lucifora et al. (2011) mostram que a criação de áreas protegidas e com restrição de pesca não é o suficiente para a conservação de elasmobrânquios, pois acreditam que, para que a conservação efetiva ocorra, seriam necessárias extensões extremamente grandes, especialmente em áreas oceânicas, o que implicaria em possíveis problemas de gestão.

O conjunto de objetivos específicos inseridos no PAN-Tubarões poderia ser uma alternativa mais viável, além das áreas de exclusão de pesca, como boas práticas de manipulação das espécies ameaçadas capturadas e soltura dos animais ainda vivos, maior controle e monitoramento das pescarias e cadeia produtiva, alternativas tecnológicas que mitiguem as capturas e mortalidade de espécies ameaçadas, além da educação ambiental. A criação de áreas marinhas protegidas globais pode ser insuficiente. Mesmo as áreas de hotspots que abrangem somente 12 países e englobariam 53 espécies endêmicas de Chondrichthyes ameaçadas, existe a complexidade de cada região ter seus planos de conservação e gestão da pesca e, devido às diversas situações geopolíticas, ocorre a dificuldade da consolidação da conservação (Davidson \& Dulvy, 2017). A conservação e o manejo efetivo de elasmobrânquios são alcançados por meio de capacitação de gestores, pesquisa, educação e divulgação (Harrison \& Dulvy, 2014). Dessa forma, o conhecimento local das comunidades estudadas pode contribuir para a criação de áreas e épocas do ano restritas à pesca de espécies de raias e tubarões ameaçados de extinção, além de agregar conhecimento sobre espécies que não são mais recorrentes na região e assim pouco estudadas e que, por isso, precisam ser divulgadas em campanhas educativas.

A respeito da importância ecológica dos elasmobrânquios, as percepções dos pescadores estão de acordo com o que pesquisadores descrevem sobre esse grupo (Dulvy et al., 2014). Quando os pescadores dizem que as raias e os tubarões são importantes para a manutenção do equilíbrio marinho e são considerados lixeiros do mar, corroboram com Jones e Geen (1977) e Camhi et al. (1998), os quais afirmam quanto ao alto nível de importância desse grupo, uma vez que ocupam o topo da cadeia trófica e participam de maneira marcante na troca de energia no ambiente em que vivem, sobretudo aqueles de regiões tropicais e subtropicais.

O CEL dos pescadores artesanais trouxe novas informações acerca dos elasmobrânquios marinhos para a região da APA do Delta do Parnaíba, da interação com a pesca local 
e sobre a conservação desse grupo, até então pouco conhecidas na região estudada. Além disso, mostrou semelhanças ao conhecimento encontrado na literatura científica e às observações realizadas em campo. Para que as ações de manejo e conservação dos elasmobrânquios sejam efetivas na APA do Delta do Parnaíba, é fundamental o trabalho permanente nas comunidades pesqueiras. $\mathrm{O}$ envolvimento das comunidades tradicionais pode contribuir com informações em longo prazo sobre as espécies ameaçadas e de difícil acesso, além de ser importante no processo de gestão participativa da biodiversidade e a preservação do ambiente para usufruto das gerações futuras.

\section{REFERÊNCIAS BIBLIOGRÁFICAS}

Abreu, J.S.; Domit, C. \& Zappes, C.A. Is there dialogue between researchers and traditional community members? The importance of integration between traditional knowledge and scientific knowledge to coastal management. Ocean E Coastal Management , 141, p. 10-19, 2017.

Alarcon, D.T. \& Schiavetti, A. O conhecimento dos pescadores artesanais de Itacaré sobre a fauna de vertebrados (não peixes) associados às atividades pesqueiras. Revista Gerenciamento Costeiro Integrado, v. 4, n. 3, 4 p., 2005.

Albuquerque, U.P. \& Lucena, R.F.P. de. Métodos e técnicas na pesquisa etnobotânica. Recife: Livro Rápido/NUPEEA, 189 p., 2004.

Almeida, Z.S. Dinâmica populacional de elasmobrânquios na costa do Maranhão. São Luís. (Relatório). 1998.

Almeida, Z.S. Levantamento e ocorrência de elasmobrânquios capturados pela pesca artesanal no litoral do Maranhão. Boletim SBBEL, v. 4, n. 10, 1999.

Araújo, C.M.E. \& Almeida, Z.S. Caracterização morfométrica de Dasyatisguttata (Bloch \& Schneider, 1801; Elasmobranchi, Dasyatidade) em águas rasas maranhenses. Pesquisa em Foco, v. 9, n. 14, p. 7-9, 2001.

Barbini, S.A. Ecologia trófica de lasrayas (Chondrichthyes, Rajidae) em el ecossistema costeiro bonaerense y uruguayo. 185 f. Ph.D. Thesis, Universidade Nacional de Mar Del Plata, Mar Del Plata. 2011.

Barbosa-Filho, M.L.V.; Schavietti; A., Alarcon, D.T. \& Costa-Neto, E.M. Shark is the man! Etnoknowledge of Brazil's South Bahia fishrmen regarding shark behaviors. J. Ethnobiol. Ethnomed., v. 10, n. 54, p. 1-14, 2014.

Batista, V.S.; Fabré, N.N.; Malhado, A.C.M. \& Ladle, R.J. Tropical ArtisanalCoastalFisheries: Challengesand Future Directions. Reviews in Fisheries Science ${ }^{\mathrm{JCR}}$, v. 22, p. 1-15, 2014.

Baum, J.; Clarke, S.; Domingo, A.; Ducrocq, M.; Lamónaca, A.F.; Gaibor, N.; Graham, R.; Jorgensen, S.; Kotas, J.E; Medina, E.; Martinez-Ortiz, J.; Monzini Taccone Di Sitizano, J.; Morales, M.R; Navarro, S.S; Pérez-Jiménez, J.C. \& Ruiz, C. Smith, W., Valenti, S.V \&Vooren, C.M. 2007. Sphyrna lewini.Sphyrna lewini.The IUCN Red List of Threatened Species 2007: e.T39385A10190088. disponível em: <http://dx.doi.org/10.2305/IUCN.UK.2007.RLTS. T39385A10190088.en.> . Acesso em: 23 jan. 2017.

Begossi, A. The fishers and buyers from Búzios Island (Brazil): kin ties and modes of production. Ciência e Cultura, v. 48, n. 3, p. 142-148, 1996. 
BegossI, A. (Org.). Ecologia de pescadores da Mata Atlântica e da Amazônia. São Paulo: Hucitec; Nepam/Unicamp; Nupaub/USP; Fapesp, 2004.

Berkes, F. Sacred ecology: tradicional ecological knowledge and resource management. Taylor and Francis, Philadelphia, Pennsylvania, USA. 209 p., 1999.

Bigelow, H.B. \& Schroeder, W.C. Fishes of the western North Atlantic. N. 1, Part. 2. Saw fishes, guitarfishes, skates, rays, and chimaeroids. Sears Foundation for Marine Research. Yale Univ., New Haven, CT. 1953.

Begossi, A. Escalas, economia ecológica e a conservação da biodiversidade. In (Org.) Meio ambiente, desenvolvimento sustentável e políticas públicas. São Paulo/Recife: Cortez/Fundação Joaquim Nabuco, 1999.

Bender, M.G.; Floeter, S.R.; Ferreira, C.E.L. \& Hanazaki, N. Mismatches between global, national and local red lists and their consequences for Brazilian reef fish conservation. Endangered Species Research, v. 18, n. 3, p. 247-254, 2012.

Camhi, M.; S.L. Fowler, J.A.; Musick, A.; Brautigan \& Fordham, S.V. Occasional paper of the IUCN Species Survival Commission, Sharks and their relatives. Ecologyandconservation, p. 1-39, 1998.

Castello, L. Repensando o estudo e o manejo da pesca no Brasil. Panam. J. Aquat. Sci., v. 3, p. 17-22, 2008.

Davidson, L.N.K. \& Dulvy, N.K. Global marine protected areas to prevent extinctions. Nat. Eco. Evol., v. 1, 0040, 2017.

Dias Neto, D. (Org.). Proposta de plano nacional de gestão para o uso sustentável de elasmobrânquios sobre-explotados ou ameaçados de sobre-explotação no Brasil. IBAMA, 156 p., 2011.

Diegues, A.C. Etnoconservação: novos rumos para a proteção da natureza nos trópicos. NUPAUB: São Paulo, 2000.

Diegues, A.C. \& Moreira, A.C.C. (Orgs.). Espaços e recursos naturais de uso comum. São Paulo: NUPAUB-USP, 2001.

Diegues, A.C. A pesca construindo sociedades. São Paulo: Núcleo de Apoio à Pesquisa sobre Populações Humanas e Áreas Úmidas Brasileiras/USP. 315 p., 2004.

Dulvy, N.K.; Fowler, S.L.; Musick, J.A.; Cavanagh, R.D.; Kyne, P.M.; Harrison, L.R.; Carlson, J.K.; Davidson, L.N.K.; Fordham, S.V.; Francis, M.P.; Pollock, C.M.; Simpfendorfer, C.A.; Burgess, G.H.; Carpenter, K.E.; Compagno, L.J.V.; Ebert, D.A.; Gibson, C.; Heupel, M.R.; Livingstone, S.R.; Sanciangco, J.C.; Stevens, J.D.; Valenti, S. \& White, W.T. 2014. Extinction riskandconservationoftheworld'ssharksandrays. 2014. Disponível em: <http://dx.doi.org/10.7554/ eLife.00590>. Acesso em: 5 dez. 2017.

Dulvy, N.K.; Simpfendorfer, C.A.; Davidson, L.N.K.; Fordham, S.V.; Brautigam, A.; Sant, G. \& Welch, D.J. Challenges and Priorities, in Sharkand Ray Conservation. Current Biology, 27, R565-R572, 2017.

Farias, A.C.S.; Fonteles-Filho, A.A.; Corrêa, C.T.C.I.; Fernandes, C.A.F. \& Cunha, F. E.A. Cadeia produtiva da pesca no interior do Delta do Parnaíba e área marinha adjacente. Fortaleza: RDS, 2015.

Feitosa, L.M.; Martins, A.P.B. \& Nunes, J.L.S. Sawfish (Pristidae) records along the Eastern Amazon coast. Endangered Species Research, v. 34, p. 229-234, 2017. 
Freitas, R.R. de.; Chamy, P. \& Dumith, R.C. Institutional design of small-scale fisheries in marine protected areas applied to sustainable territorial development on the Brazilian coast. Ocean E Coastal Management, 139, p. 92-101, 2017.

Gadig, O.B.; Bezerra, M.A.; Feitosa, R.D. \& Furtado-Neto, M.A.A. Ictiofauna marinha do estado do Ceará, Brasil: I. Elasmobranquii. Arq. Ciên. Mar, Fortaleza, v. 33, p. 127-13, 2000.

Giglio, V.J. \& H. Bornatowski. Fishers' ecological knowledge of smalleye hammerhead, Sphyrna tudes, in a tropical estuary. Neotropical Ichthyology, v. 14, n. 2, 150103, 2016.

Harrison, L.R. \& Dulvy, N.K. (Eds.). Sawfish: a Global Strategy for Conservation. IUCN Species Survival Commission's Shark Specialist Group, Vancouver, Canada. 2014.

Heithaus, M.R.; Frid, A.; Wirsing, A.J.; Dill, L.M.; Fourqurean, J.W.; Burkholder, D.; Thomson, J.A. \& Bejder, L. State-dependent risk-taking by green sea turtles mediates topdown effects of tiger shark intimidation in a marine ecosystem. Journal of Animal Ecology, 76, p. 837-844, 2007.

ICMBio. Instituto Chico Mendes. Avaliação do risco de extinção dos elasmobrânquios e quimeras no Brasil: 2010-2012. 20016. Disponível em: <http://www.icmbio.gov.br/cepsul/images/stories/ biblioteca/download/trabalhos_tecnicos/pub_2016_avaliacao_elasmo_2010_2012.pdf>.

ICMBio. Instituto Chico Mendes. Caracterização da unidade e temas complementares: Reserva Extrativista Marinha do Delta do Parnaíba. Brasília, 2009.

Jones, B.C. \& Geen, G.H. Reproduction and embryonic development of spiny dogfish (Squalus acanthias), in The Strait of Georgia, British Columbia. Journal of Fisheries Research Board Canada, Vancouver, 34, p. 1286-1292, 1977.

Kotas, J.E.; Petrere Jr., M.; Dos Santos, R.A.; Bustamante, A.; Lin, C.F.; Menezes, A.A.S. \& Micheletti, E.L.V. The horizontal migration of hammerhead sharks along the southern Brazilian coast, based on their exploitation pattern and considerations about the impact of anchored gillnets activities on these species. Revista CEPSUL - Biodiversidade e Conservação Marinha, v. 3, n. 1, p. 45-68, 2012.

Kyne, P.M.; Ishihara, H.; Dudley, J.S.F. \& White, W.T. 2006. Aetobatus narinari. Lista Vermelha da IUCN de Espécies Ameaçadas 2006: e.T39415A10231645. Disponível em: <http://dx.doi.org/10.2305/IUCN.UK.2006.RLTS.T39415A10231645.en>. Acesso em: 23 jan. 2017.

Lessa, R.P.T. Levantamento faunístico dos elasmobrânquios (Pisces, Chondrichthyes) do litoral ocidental do estado do Maranhão, Brasil. Boletim do Laboratório de Hidrobiologia, 7, p. 27-41, 1986.

Lessa, R.; Charvet-Almeida, P.; Santana, F.M. \& Almeida, Z. 2006. Isogomphodon oxyrhynchus. The IUCN Red List of Threatened Species 2006: e.T60218A12323498. Disponível em: <http:// dx.doi.org/10.2305/IUCN.UK.2006.RLTS.T6018A12323498.en.>. Acesso em: $1^{\circ}$ jun. 2018.

Lessa, R.; Quijano, S.M; Santana, F.M. \& Monzini, J. 2006. Rhizoprionodon porosus. The IUCN Red List of Threatened Species 2006: e.T61407A12473033. disponível em: <http://dx.doi. org/10.2305/IUCN.UK.2006.RLTS.T61407A12473033.en> . Acesso em: 23 jan. 2017.

Lucifora, L.O.; Garci 'A, V. B. \& Worm, B. Global Diversity Hotspots and Conservation Priorities for Sharks. PLoS ONE, v. 6, n. 5, 2011.

Machado, M.R.B.; Almeida, Z. da S. De Da \& Castro, A.C.L. Estudo da biologia reprodutiva de Rhizoprionodon porosus Poey, 1861 (Chondrychthyes: Carcharhinidae) na plataforma 
continental do estado do Maranhão, Brasil. Boletim do Laboratório de Hidrobiologia, São Luís, 13, p. 51-65, 2000.

Martins, A.P.B.; Feitosa, L.M.; Lessa, R.P.; Almeida, Z.S.; Heupel, M.; Silva, W.M. Analysis of the supply chain and conservation status of sharks (Elasmobranchii: Superorder Selachimorpha) based on fisher knowledge. PLoS ONE, 13, n. 3: e0193969. 2018. Disponível em: https://doi.org/10.1371/ journal.pone.0193969.

Marques, J.G.W. Pescando pescadores: ciência e etnociência em uma perspectiva ecológica. 2. ed., São Paulo, Brasil: NUPAUB/ USP, 304 p., 1995.

Mendonça, F.F.; Oliveira, C.; Gadig, O.B. \& Foresti, F. Phylogeography and genetic population structure Rhizoprionodon porosus. Rev. Fish. Biol. Fisheries, 21, p. 799-814, 2011.

Oliveira Jr., J.G.C.; Silva, L.P.S.; Malhado, A.C.M.; Batista, V.S.; Fabré, N.N. \& Ladle, R.J. Artisanal Fisheries Research: a Need for Globalization? Plos One, v. 11, p. e0150689, 2016.

O'Riordan, T. \& Turner, R.K. (Eds.). Growth and resource depletion, in Annotated reader in environmental planning and management. Great Britain: Pergamon International Library, p. 322-345, 1997.

Paz, V.A. \& Begossi, A. Ethnoichthyology of gamboa fishermen of Sepetiba Bay, Brazil. J. Ethnobiol., v. 16, n. 2, p. 157-158, 1996.

Posey, D. Interpreting and applying the reality of indigenous concepts: what is necessary to learn from the natives. In: Redford K., Padoch C, editors. Conservation in Neotropical Forests: Working from Traditional Resource Use. Columbia University Press.1992.

Reis-Filho, J. A.; Freitas, R.H.A.; Loiola, M.; Leite, L. and others. Traditional fisher perceptions on the regional disappearance of the largetooth sawfish Pristis pristis from the central coast of Brazil. Endang Espécies Res. 29, p. 189-200, 2016.

Rosa, R.S; Castro, A.L.F; Furtado, M.; Monzini, J. \& Grubbs, R.D. 2006. Ginglymostoma cirratum. The IUCN Red List of Threatened Species 2006:e.T60223A12325895. Disponível em: <http://dx.doi.org/10.2305/IUCN.UK.2006.RLTS.T60223A12325895.en>. Acesso em: 23 jan. 2017.

Sáenz-Arroyo, A. \& Revollo-Fernández, D. Local ecological knowledge concurs with fi shingstatistics: an example from the abalone fi shery in Baja California, Mexico. Marine Policy, v. 71, p. 217-221, 2016.

Simpfendorfer, C.A. \& Wiley, T.R. National smalltooth sawfish encouter database. Mote Marine Laboratory Technical Report 1134. A final report for NOAA Purchase Order No. GA13F05SE5547. 13 p., 2006.

Schalaff, A.M.; Heupel, M.R.; Simpfendorfer, C.A. Influence of environmetal factors on shark and ray movement, behavior and habitat use: review. Rev. Fish Biol. Fish. 24, 10891103, 2014.

Tagliafico, A.; Rago, N.; Rangel, S. \& Mendoza, J. Exploitation and reproduction of the spotted eagle ray (Aetobatus narinari) in the Los Frailes Archipelago, Venezuela. Fishery Bulletin, 110, p. 307-316, 2012.

Turner, R.K.; Georgiou, S.; Brouwer, R.; Bateman, I.J. \& Langford, I.J. Towards an integrated environmental assessment for wetland and catchment management. Geographical Journal, 169, p. 99-116, 2003. 
Uvo, C.R.B. \& Nobre, C.A. A Zona de Convergência Intertropical (ZCIT) e a precipitação no norte do Nordeste do Brasil. Parte I: a posição da ZCIT no Atlântico Equatorial. Climanalise, v. 4, n. 07, p. 34-40, 1989.

Vooren, C.M. \& Klippel, S. Ações para a conservação de tubarões e raias no sul do Brasil. Porto Alegre, Igaré, 262 p., 2005.

Yokota, L. \& Lessa, R.P. Reproductive biology of three ray species: Gymnura micrura (Bloch \& Schneider, 1801), Dasyatis guttata (Bloch \& Schneider, 1801) and Dasyatis marianae Gomes, Rosa \& Gadig, 2000, caught by artisanal fisheries in Northeastern Brazil. Cah. Biol. Mar. 48, p. 249-257, 2007. 